\title{
Mecanismos y dinámicas del trabajo en las plataformas digitales: los casos de Airbnb y de las plataformas de reparto
}

\author{
Mechanisms and dynamics of work on digital platforms: the \\ cases of Airbnb and delivery platforms
}

\author{
Francisco José Fernández-Trujillo Moares \\ Universidad Nacional de Educación a Distancia \\ franciscoftm@poli.uned.es (ESPAÑA)
}

\author{
Javier Gil García
}

Uppsala University

javier.gil@ibf.uu.se (SUECIA)

Recibido: 16.11 .2020

Aceptado: 23.08 .2021

\section{RESUMEN}

El presente artículo analiza las formas de trabajo y de modos de producción que configuran las plataformas digitales. Se plantea que en estas pueden emerger distintas formas de trabajo, pero que todas ellas responden a las transformaciones del neoliberalismo contemporáneo. Las formas en las que se dan sus configuraciones y las de sus procesos productivos, impulsan nuevas formas laborales y de producción que rompen con los modelos estándar para generar nuevas vías de producir plusvalías. El artículo se centra en los casos de Airbnb y diversas plataformas de reparto de comida y mensajería. Alguna de las características que se observan es que se trata de un tipo de trabajo invisibilizado, empresarial, que surge sobre lo reproductivo, que se articula sobre tiempo de trabajo no pagado, que impulsa dinámicas de individualización y aislamiento en el desarrollo de esta actividad o que genera una paradójica relación entre la sensación de autonomía y libertad como autónomos contrapuesta a un fuerte despliegue de mecanismos de control y organización del trabajo. Para llevar a cabo esta investigación se han realizado entrevistas abiertas semi-estructuradas a anfitriones de Airbnb y repartidores de Deliveroo, Glovo, Uber Eats y Stuart. 


\title{
PALABRAS CLAVE
}

Capitalismo de plataforma, neoliberalismo, trabajo, Airbnb, plataformas de reparto.

\begin{abstract}
The present paper analyses the ways of working and modes of production that configure digital platforms. It argues that different forms of work can emerge in these, but that all of them respond to the transformations of contemporary neoliberalism. The forms in which their configurations and those of their productive processes are given, promote new forms of work and production that break with the standard models to generate new ways of producing surplus value. The paper focuses on the cases of Airbnb and various food delivery and messenger platforms. Some of the characteristics that can be observed are that it is a type of invisible, entrepreneurial work that arises from the reproductive aspect, that is articulated on the basis of unpaid work time, that promotes dynamics of individualization and isolation in the development of this activity or that generates a paradoxical relationship between the feelings of autonomy and freedom as self-employed workers as opposed to a strong deployment of mechanisms of control and organization of work. In order to carry out this research, open semistructured interviews were conducted with Airbnb hosts and Deliveroo, Glovo, Uber Eats and Stuart deliverers.
\end{abstract}

\section{KEY WORDS}

Platform capitalism, neoliberalism, labour, Airbnb, delivery platforms

\section{INTRODUCCIÓN}

Desde el nacimiento de las economías colaborativas sus impulsores han anunciado todos los efectos positivos que estas economías tendrían (Botsman, 2010; Eckhardt et. Al, 2019). Uno de los elementos principales de estos discursos son las posibilidades que generan a los particulares para ofrecer servicios que les permitan obtener ingresos extra de manera flexible (Comisión Europea, 2016; Schor, 2017; Zervas et al, 2014). Estas plataformas estarían permitiendo a las personas recuperar parte de la capacidad adquisitiva perdida durante la crisis (Gottlieb, 2013:201; Schor y Attwood-Charles, 2017; Sperling, 2015), siendo estas posibilidades muy importantes para quienes están en situaciones de mayor vulnerabilidad económica y social (Quattrone et al., 2016; Schor, 2017). Por lo tanto, las economías colaborativas estarían fomentando un mayor reparto de los 
activos y creando nuevas oportunidades de redistribución económica (Comisión Europea, 2016; Sundararajan, 2016).

Sin embargo, las plataformas digitales han recibido numerosas y variadas críticas. Diversos trabajos señalan que la actividad que promueven no tiene nada que ver con "compartir" y que en realidad se trata de alquilar, vender y responder a intereses personales (Frenken y Schor, 2017; Oskam y Boswijk, 2016; Ravenelle, 2017; Schor, 2017; Gordo et al., 2016). Así, el eufemismo de "colaborativas" se estaría empleando como una estrategia de marketing (De Rivera et al., 2017), que facilite invisibilizar las nuevas formas de explotación laboral y de concentración de riqueza que estas plataformas generan (Degryse, 2016; Hill, 2015; Kalamar, 2013; Schor y Attwood-Charles, 2017; Scholz, 2016; Slee, 2015). Esto ha llevado a plantear que estamos ante una nueva dinámica de reproducción del capitalismo: el capitalismo de plataforma (Snircek, 2018).

En este contexto, el presente artículo analiza las formas de trabajo y de modo de producción que configuran las plataformas digitales, planteando que en estas pueden emerger distintas formas de trabajo, pero que todas ellas responden a las transformaciones del neoliberalismo contemporáneo (Brown, 2015; Gago, 2015; Laval y Dardot, 2013). En otras palabras, las configuraciones y los procesos productivos del capitalismo de plataforma impulsan nuevas formas laborales y de producción que rompen con los modelos estándar para generar nuevas vías de producir plusvalías. Es por ello que las plataformas digitales se convierten en ejemplos muy ilustrativos del tipo de trabajo que extiende el neoliberalismo en su etapa post-2008. En el artículo se analizan los casos de las plataformas Airbnb y diversas plataformas de reparto de comida y mensajería. En ambos casos se analiza cómo se organiza y configura el trabajo en las distintas plataformas.

\section{EL TRABAJO EN LAS PLATAFORMAS DIGITALES: ¿NUEVAS FORMAS DE TRABAJAR?}

Para comprender el desarrollo de estas plataformas ha de tenerse en cuenta que emergen en un contexto económico y social concreto que será determinante. Este es el de la crisis de 2008, en el que se han generado diferentes mecanismos que han supuesto modificaciones en las lógicas del trabajo estándar (Campos y Hernández, 2018). Entre estas se encontrarían las plataformas digitales, en las que su forma de desarrollo, evolución y características se podrían considerar de cierta manera producto de dicha crisis (Srnicek, 2018; Gil, 2016). Con la Gran Recesión de 2008, comienza una fase de deterioro de las condiciones laborales, de mercados laborales más precarizados, de altas tasas de desempleo y de desarrollo de diferentes formas de empleo atípico (Calderón y López Calle, 2010; Srnicek, 2018; Santos y Martín, 2012). Al mismo tiempo, se ha producido una profundización y continuación de la expansión tecnológica mediada por los datos, nuevos modelos de negocio y la reformulación de los procesos productivos (De Stefano, 2018; Veen et al., 2019; Graham et al., 2017). Estos no serían unos 
procesos espontáneos o aislados, sino una tendencia en la transformación del trabajo como resultado de las prácticas neoliberales extendidas en las últimas décadas, encaminadas al desgaste de los sistemas de protección y bienestar asociados al trabajo a través de diferentes vías (Alonso, 1999; Laval y Dardot, 2013). Entre las críticas, también cabe destacar la pérdida de derechos asociados al trabajo, especialmente en su dimensión colectiva, como la negociación o la representación (De Stefano, 2016; Vandaele, 2018).

Las plataformas son para las empresas una herramienta a través de la que organizar el trabajo (De Stefano, 2016: 5). Así, es necesario tener en cuenta que el diseño y formulación de los procesos informáticos responden a unos criterios determinados y que no están sujetos a una formulación aséptica o inocente. En otras palabras, cómo son diseñadas las aplicaciones, plataformas y procesos de producción de las plataformas responden a criterios de las empresas atendiendo a los intereses de la producción, como la productividad, ahorro, búsqueda de beneficios, competencia con otras plataformas y precedentes de prestatarios de los servicios en el mercado, etc. (De Rivera et al., 2017; Sadin, 2018). Así, algunos autores como Griesbach, Reich, Elliott-Negri y Milkman (2019) desarrollan el concepto de "despotismo algorítmico" para abordar cómo mediante la utilización de las plataformas y aplicaciones digitales, las empresas hacen responsable al proceso algorítmico de la toma de decisiones, teniendo consecuencias perjuiciosas para las condiciones y el desarrollo de la actividad de los trabajadores y las trabajadoras.

En esta investigación abordamos el tipo de trabajo que emerge en estas plataformas, y si responde a la categoría de "empleados, trabajadores autónomos u otra figura nueva" (Sundararajan, 2016: 178). Partimos de que no se puede hablar del trabajo y de los procesos productivos de las plataformas digitales como un todo homogéneo. Estos varían no solo entre plataformas, sino que además en la propia plataforma se pueden observar distintas formas de trabajo. Aun así, sí que se observan tendencias determinadas, sobre todo en el tipo de trabajo que configuran las plataformas con mayor volumen e implantación, como pueden ser Airbnb, Uber, Deliveroo o Glovo.

El trabajo en las plataformas digitales configura actividades laborales tradicionales que ahora se organizan por medio de la plataforma (De Stefano, 2016; Miguélez, 2018; Srnicek, 2018), dándose algunos mecanismos que los hace particulares y los sitúa en formas atípicas de trabajo. El trabajo en las plataformas digitales se ha implantado en diferentes ámbitos de la economía y ha adoptado distintas formas, con características concretas dependiendo del sector al que se atienda (Drahokoupil y Jepsen, 2017; Graham et al., 2017). Estas pueden ser categorizadas de diferentes maneras según distintos criterios como actividad; relación entre usuario, plataforma y quienes prestan los servicios; cualificación; o tipos de tareas, entre otras (Florisson y Mandl, 2018; Howcroft. y BergvallKåreborn, 2019; Vallas, 2019). 
El concepto de "prosumición" también ha sido utilizado para explicar el tipo de trabajo que emerge en estas plataformas como un trabajo que no responde a las categorías tradicionales (Denegri-Knott y Zwick 2012; Rey, 2012; Humphreys y Grayson, 2008; Rifking, 2014). Las innovaciones tecnológicas de las últimas décadas han generado procesos que hacen que la división entre productores y consumidores se diluya, surgiendo el sujeto prosumidor como aquel que realiza funciones de producción y consumo de forma simultánea. El elemento principal es que los consumidores se incorporan a los procesos de producción (ofreciendo servicios en Airbnb o Blablacar), mientras que el consumo se vuelve productivo (escribiendo evaluaciones en Amazon o Yelp, o utilizando Facebook o Twitter). De esta manera producción y consumo ya no aparecen como dos esferas separadas: cada vez más la producción se genera en espacios de consumo, mientras que el consumo se hace más productivo (Rey, 2012). Algunos autores han planteado que la prosumición es en sí misma una forma de extender procesos de explotación y de trabajo no remunerado en nuestras sociedades (Fuchs, 2010; Humphreys y Grayson, 2008; Ritzer y Jurgenson, 2010; Comor, 2010), configurando en muchos casos nuevas formas de trabajo no remunerado o free labour (Fast et al., 2016; Terranova, 2000). En relación a las plataformas digitales donde se ofrecen servicios (como Airbnb), este concepto se podría aplicar a los casos de los usuarios que ofrecen servicios de manera esporádica en la plataforma, de forma no profesionalizada (Gil, 2018).

Como se observa, la aparición y el desarrollo de las plataformas digitales en el marco de la transformación del trabajo implica nuevos debates. Uno de ellos es el debate jurídico y legislativo a propósito de la conveniencia de generar un marco regulatorio específico para estas formas de trabajo crecientes o el acomodo a los ya existentes (Körfer y Röthig, 2017; Todolí-Signes, 2017). En ese sentido, los resultados de investigaciones empíricas como las que se presentan en este artículo son necesarias también para avanzar en el campo jurídico y legislativo de las plataformas digitales, pero también en el resto de debates que se vienen generando a propósito de otros aspectos. En este sentido, es de relevancia puntualizar que estos análisis se hicieron antes del desarrollo y aprobación del Real Decreto-ley 9/2021 (popularmente conocido como ley Rider) que, al menos parcialmente, regula en España desde el 12 de agosto de 2021 la actividad laboral del reparto en el ámbito de plataformas digitales. El desarrollo de esta normativa pone en cuestión y modificará seguramente algunas de las dinámicas que aquí se abordan. Sin embargo, tenemos que tener en cuenta que estas dinámicas están presentes a nivel global y se están dando en otros sectores donde que la citada ley no ampara. Igualmente, como es analizado en este artículo, observamos que las dinámicas y lógicas presentes en las plataformas digitales están sujetas a cambios y cuentan con una gran capacidad de adaptación. Es por esto, que estos análisis son de una total vigencia. 


\section{METODOLOGÍA}

En este artículo se analizan los datos extraídos de dos estudios originales y separados llevados a cabo por los autores — uno sobre la plataforma Airbnb y otro sobre las plataformas reparto de comida y mensajería-. Ambos proyectos analizan los tipos de trabajo que emergen en estas plataformas, en relación a factores estructurales como la crisis, las transformaciones del neoliberalismo, la precariedad laboral y los mercados de trabajo. El análisis comparado nos permite ampliar inferencias sobre el tipo de trabajo que emerge en estas plataformas y cómo se configuran los procesos productivos en estas, observando similitudes y diferencias que nos permitan ahondar en estos nuevísimos fenómenos sociales. Asimismo, la propuesta metodológica para ambas investigaciones han sido las entrevistas abiertas. Para ello se ha recurrido a entrevistas semi-estructuradas que se han diseñado, realizado y analizado partiendo de la línea teórico-práctica de la denominada Escuela Cualitativa Madrileña (Alonso, 1994, 1998; Alonso et al., 2016; Arribas, 2013; Callejo, 2001; Conde y del Álamo, 2009; Criado, 1998, 2014; Ibáñez, 1979, 1986, 1991).

En el caso de Airbnb, las entrevistas se realizaron en las ciudades de Nueva York, San Francisco, Madrid y Barcelona entre 2015 y 2019. Se efectuaron un total de 55 entrevistas a anfitriones en las cuatro ciudades. En el caso de las plataformas de reparto, se ha entrevistado a 40 repartidores de Madrid, Barcelona, Sevilla, Bilbao, Cádiz y Valencia, que han trabajado en una o varias de las grandes plataformas de reparto (Deliveroo, Glovo, Uber Eats y Stuart). En este caso, el trabajo de campo se ha realizado entre marzo de 2019 y abril de 2020. Estas entrevistas han atendido a criterios de variabilidad de las variables sociológicas típicas, como género, edad, origen, etc.

A pesar de ser dos investigaciones independientes, este estudio permite observar que se trata de dinámicas que, aunque no sean iguales, pertenecen a una misma tendencia que se da en un mismo sentido y que utilizan las plataformas como herramienta para ello. A diferencia de otros procesos al margen de las plataformas -que también se están extendiendo-, estas generan una neoliberalización del trabajo en diferentes sentidos (individualización, desregulación y desreglamentación, inseguridad, desvanecimiento de las fronteras entre trabajo y vida cotidiana, etc.). Por ello, la comparación de dos ámbitos nos permite analizar con mayor profundidad la extensión de estas transformaciones.

\section{EL CASO DE AIRBNB}

La plataforma Airbnb nace en el año 2007 en la ciudad de San Francisco. Brian Chesky y Joe Gebbia, dos jóvenes diseñadores, iban a perder su piso el día que su casero les comunicó que les subía el alquiler. Aprovechando que en San Francisco se iba a celebrar un congreso de diseñadores, que todos los hoteles estaban completos y que disponían de tres colchones hinchables, decidieron ofrecer alojamiento en su salón a tres personas que vinieran al congreso. Su oferta de 
hospedaje incluía dormir en los colchones hinchables, el desayuno, una tarjeta de transporte y monedas sueltas para dar a las personas sin hogar de la ciudad. La idea funcionó y durante los días del congreso tres personas se alojaron en su vivienda pagando $80 \$$ al día.

La actividad de Airbnb responde al modelo del "hospedaje colaborativo", y se ha convertido en un referente e icono de las economías colaborativas a nivel internacional. A día de hoy Airbnb cuenta con más de 5 millones de alojamientos y tiene más hospedajes a nivel internacional que las cinco cadenas hoteleras más grandes del mundo juntas (Hartmans, 2017). Está presente en 81.000 ciudades y en más de 191 países, acumula más de 400 millones de reservas y cada noche más de 2 millones de personas se alojan de media en un Airbnb (Airbnb Press Room, s. f.).

En tan solo 10 años, Airbnb se ha convertido en la empresa del mundo con más plazas de hospedaje con una plantilla de en torno a 3.100 trabajadores (Gallagher, 2017). ¿Cómo ha sido posible el crecimiento y expansión internacional de esta empresa con una fuerza de trabajo tan reducida? Este hecho solo ha sido posible a través del desarrollo de una fuerza laboral y proceso productivo con particularidades muy concretas, que rompen parcialmente con las formas de configuración del trabajo y del proceso productivo de la economía tradicional. El trabajo y el proceso productivo en Airbnb se caracterizan por: surgir sobre la esfera de la reproducción, ser un tipo de trabajo invisibilizado que no es reconocido como empleo y por adquirir la forma de trabajo empresarial.

\subsection{Trabajo que surge sobre la esfera de la reproducción}

El primer paso de los anfitriones que hospedan en su residencia, es crear y organizar el proceso productivo. El proceso productivo en Airbnb rompe con las formas en que se organiza la producción en la economía tradicional. Principalmente porque la producción no se articula sobre actividades económicas de la esfera productiva. Por el contrario, en Airbnb la producción se desarrolla sobre el campo de la reproducción y sobre actividades vitales de la persona. Las principales características del proceso productivo en Airbnb son las siguientes:

1. En Airbnb el proceso productivo se organiza desde la esfera de la reproducción para convertir el campo de la reproducción en un campo productivo. En la economía tradicional, la producción se constituye como una esfera propia,

${ }^{1}$ En Airbnb existen al menos dos modelos de hospedaje: el modelo p2p y el modelo comercial. En el modelo comercial la actividad no cumple los principios de las economías colaborativas. Se trata de alquileres de corta duración, en viviendas cuya única función es ser alquiladas en Airbnb y se realiza de forma profesional. Por el contrario, el modelo p2p es el que sí que cumple con los principios de las economías colaborativas. La característica principal de este modelo es que los anfitriones hospedan en su residencia habitual. En este artículo nos referimos en exclusiva al trabajo y proceso productivo que surge en el modelo p2p (ya que es el modelo que sí que cumple los principios de las economías colaborativas). 
donde todos los recursos, tiempos, actividades y relaciones que se movilizan tienen un fin concreto: la producción de valor. Esta esfera se constituye como una dimensión separada y propia del resto de esferas sociales. Por el contrario, en Airbnb, el proceso productivo se organiza sobre la esfera reproductiva de las personas, para convertir el campo de la reproducción en un campo productivo. La producción no tiene una esfera propia y separada del resto de esferas sociales, y por medio de la plataforma la esfera reproductiva se vuelve productiva. La actividad de Airbnb consiste en transformar recursos, actividades, relaciones, tiempos y atributos personales del ámbito de lo privado y de lo reproductivo en fuente de valor (en medio de producción).

2. El recurso que se moviliza a través de Airbnb para obtener beneficios es la propia vivienda de la persona, que es transformada en medio de producción. Este recurso es un bien de consumo, ya que la persona adquiere la vivienda - en régimen de propiedad o de alquiler - para hacer uso de la misma como hogar, como un sitio donde vivir. Por lo tanto, la vivienda cumple una función social. En cambio, en la economía tradicional, los recursos que se movilizan en la producción adquieren la forma de bienes de inversión, puesto que el objetivo de la adquisición del recurso es que produzca valor. El fin último del bien no es que su valor de uso sea consumido por la persona que lo adquiere, sino utilizarlo como medio de producción para que genere valor de cambio en el mercado. Lo novedoso de Airbnb, es que a través de la plataforma los anfitriones tienen la capacidad de transformar temporalmente bienes de consumo del campo de la reproducción en bienes de inversión que generan beneficios en el mercado. Convertir su vivienda, como bien de consumo que cumple una función social, en un bien de inversión que tiene una función económica.

3. La producción no se articula sobre actividades económicas, sino sobre actividades vitales de las personas. La motivación principal de los anfitriones es realizar actividades propias del campo de la reproducción, como irse de vacaciones. Ahora, gracias a Airbnb, emerge una segunda motivación: la producción de valor. Por ello, a través de Airbnb las personas pueden transformar elementos propios del campo de la reproducción (como su vivienda) en elementos productivos. Pero a diferencia de la economía tradicional, la producción no se articula en torno a la producción de valor, sino que, por el contrario, la producción de valor se incrusta y se supedita a la vida misma de la persona. Es decir, la producción de valor se incorpora sobre el ciclo vital de la persona. Como resultado las actividades vitales de las personas se transforman en actividades productivas.

Por ello, el tipo de proceso productivo de acumulación y de valorización que emerge sobre Airbnb se constituye como una forma desarrollada de biocapitalismo en el marco del neoliberalismo en su face post-2008 (Fumagalli, 2007). En efecto, estamos ante un modelo en el que el proceso de acumulación y de valorización se funda por completo sobre la propia vida de las personas, lo que favorece la valorización económica de la vida misma. 


\subsection{Trabajo invisibilizado}

El modelo productivo de Airbnb, al igual que el resto de plataformas de la economía de Silicon Valley, forma parte de un momento histórico en el desarrollo del capitalismo. Se trata de una forma de organización socioeconómica en que el trabajador es eliminado del aparato productivo para ser sustituido por un consumidor. Los consumidores se encargan de realizar el trabajo que antes realizaban los trabajadores.

En el caso de Airbnb, quienes realizan estas funciones son los denominados "anfitriones". Estos desarrollan toda una serie de actividades para generar un servicio de hospedaje en la plataforma, por medio de transformar su vivienda en un bien de inversión que permita la producción de valor. La característica principal de este modelo productivo y laboral, es que los anfitriones no son reconocidos como trabajadores desde una perspectiva económica, política, jurídica, social ni simbólica. Por lo tanto, tampoco cuentan con los derechos ni garantías que tienen los trabajadores en la economía tradicional ni con los deberes tributarios.

Este hecho es un elemento principal del modelo laboral de Airbnb: que su fuerza de trabajo, aquella que genera dos millones de hospedajes al día a nivel internacional, no sea reconocida como fuerza de trabajo. Para la empresa, los anfitriones no son trabajadores, sino personas que reciben a huéspedes en sus hogares casi de forma altruista (aunque cobren por ello), motivados por la ilusión de compartir, de conocer a personas de otras culturas y de desarrollar relaciones afectivas, como se observa en sus publicidad (Airbnb, 2016a; 2016b). De hecho, la empresa utiliza todo tipo de eufemismos para no reconocer a los anfitriones como trabajadores, denominándoles "colaboradores", "embajadores de la ciudad”, etc (Degryse, 2016; Gil, 2019).

Pero la percepción de los anfitriones de Airbnb es completamente opuesta a la publicidad y discursos de la empresa. Absolutamente todos los anfitriones entrevistados reconocen que la actividad de ofrecer servicios en la plataforma es una forma de trabajo. Para algunos se trata sin lugar a duda de un trabajo, otros consideran que es un tipo de trabajo particular, algunos piensan que adquiere la forma de trabajo y otros consideran que no solo es trabajo, sino que es mucho trabajo. Pero en ningún caso los anfitriones consideran que la actividad de Airbnb sea ajena al campo laboral.

El hecho de que sea un tipo de trabajo que emerge sobre la esfera de la reproducción, facilita que este tipo de trabajo sea invisibilizado por la empresa. La producción se organiza sobre la propia vida, recursos, actividades y relaciones de los anfitriones, que se hacen productivas, lo que hace que Airbnb pueda justificar que estas actividades no tienen que ser consideradas como formas de trabajo. Porque para la empresa, en última instancia, lo que hacen los anfitriones es recibir a huéspedes en su vivienda a cambio de un ingreso.

Airbnb también ha desarrollado mecanismos de control y disciplinamiento del trabajo de los anfitriones, que también son invisibilizados. Se trata de dispositivos que no ejercen un control directo sobre el trabajo, pero que fuerzan a los anfitriones a comportarse y a producir de una determinada manera. El principal 
mecanismo es el sistema de reputación digital, que permite que los huéspedes evalúen a los anfitriones atendiendo a distintos criterios como la comunicación o la limpieza de la vivienda. Si los anfitriones son evaluados de forma negativa, será muy difícil que puedan seguir hospedando en la plataforma a largo plazo. Otros elementos que incluye la plataforma, como el tiempo de respuesta de los anfitriones a las solicitudes o mensajes de los huéspedes, también impulsan un tipo de comportamiento determinado sobre los anfitriones. De esta manera, se observa como el diseño de la plataforma y del sistema de reputación digital cumplen el objetivo de disciplinar de una determinada manera a la fuerza de trabajo.

El tipo de trabajo invisibilizado que emerge en Airbnb, se constituye por las pautas actuales de bioproducción en el contexto histórico del biocapitalismo (Fumagalli, 2007; Lara, 2013). Este se caracteriza por configurar un sistema en que la producción de valor se extrae de la vida misma. Un modelo que no consisten invertir en capital constante ni capital variable, sino en invertir "en dispositivos de producción y captación del valor producido en el exterior de los procesos reconocidos formalmente como productivos" (Lara, 2013: 602). El plusvalor se genera principalmente "del ahorro de los costes del trabajo en la medida en que este resulta externalizado" (602) sobre los anfitriones. Además, la producción de los anfitriones reside en la explotación integral "del individuo social" (602-603), ya que la producción coloniza el conjunto de las facultades genéricas, lingüísticas, emocionales y relacionales de los anfitriones.

\subsection{Trabajo empresarial}

El trabajo de los anfitriones de Airbnb se constituye como una forma de trabajo empresarial. Los anfitriones no solo producen una oferta de hospedaje, sino que son los encargados de organizar el conjunto del proceso productivo y de gestionar la oferta como si de una empresa se tratara.

En Airbnb son los anfitriones y no la empresa los encargados de organizar el conjunto de la producción y de configurar su actividad como una forma de trabajo. Por un lado, se encargan de producir el servicio: acondicionar el espacio, la limpieza, la entrega de llaves y la comunicación con los huéspedes. Por otro, se encargan de hacer que la oferta sea competitiva y asumen todos riesgos de la actividad. Para ello, dotan de valor a la oferta a través de distintas formas (principalmente elementos lingüísticos y comunicativos) y analizan los precios de la ciudad y de su barrio para fijar el suyo propio, que cambian de forma flexible según el mercado.

Para aumentar la competitividad y rentabilidad de su oferta, los anfitriones también impulsan procesos de externalización, automatización y división del trabajo. Los procesos de división del trabajo se suelen ejecutar cuando los anfitriones son más de una persona (una pareja o compañeros de piso). En estos casos se dividen las actividades productivas de la forma en que estas generen menos costes a los anfitriones. Por ejemplo, será frecuente que los anfitriones con horarios laborales flexibles o que trabajan desde casa se encarguen de la entrega de 
llaves, o que aquellos más dispuestos a comunicarse con desconocidos o utilizar el móvil frecuentemente, se encarguen de la comunicación. También habrá actividades que los anfitriones no deseen hacer, y por lo tanto las externalizarán.

La externalización del trabajo de los anfitriones adquiere distintas formas. En algunos casos es completa (todas las actividades se externalizan sobre otra persona o empresa que se encarga de realizar todas las funciones productivas: limpieza, entrega de llaves, comunicarse con los huéspedes, etc). En otros, solo se externalizan determinadas funciones que los anfitriones no desean realizar (como la limpieza). La externalización se puede realizar sobre profesionales, ya sea empresas especializadas o personas a las que se contrata. Pero también se puede externalizar sobre relaciones sociales no económicas, basadas en vínculos personales, relaciones de confianza, de vecindario o de amistad. Actividades como la entrega de llaves, se solicita a un familiar o amigo que se encargue, o directamente se dejan en un comercio cercano a la vivienda. También se suele recurrir a esta forma de externalización cuando surge un improvisto y los anfitriones no pueden gestionarlo (porque no estén en la ciudad o se encuentren en su trabajo). Una característica de esta forma de externalización es que por lo general no se remunera el trabajo de las personas implicadas, aunque desempeñen una función principal en la cadena productiva.

Los anfitriones también racionalizan su trabajo por medio de automatizar algunos elementos de la cadena productiva, lo que reduce los costes y los tiempos de su trabajo. Un elemento de automatización es la entrega de llaves a los huéspedes. Recibir a los huéspedes es una de las actividades que más coste genera a los anfitriones, al tener que adaptarse a los horarios de los huéspedes y asumir los tiempos de espera (que pueden llegar a ser elevados). Por ello muchos anfitriones optan por poner candados con la llave de la vivienda. Una vez los huéspedes hacen la reserva, los anfitriones les envían el código del candado y cuando estos llegan a la vivienda, lo introducen para acceder a la llave. Otro elemento de automatización es la "reserva instantánea". Esta opción reduce los tiempos de comunicación entre anfitriones y huéspedes, aunque aumenta los riesgos para los anfitriones (ya que normalmente les gusta cerciorarse del perfil de persona que van a recibir en su vivienda). Otros elementos son el uso de programas de "Smart pricing", que fijan los precios del servicio según las condiciones del mercado, o de "respuesta automática", que contestan directamente a las solicitudes y mensajes de los huéspedes.

\section{EL CASO DE LAS PLATAFORMA DE REPARTO DE COMIDA Y MENSAJERÍA}

En el año 2015 empezaba a funcionar en España Deliveroo, dos años después de su fundación en Reino Unido. En 2014 tendría lugar la fundación de Glovo en Barcelona, que pronto se extendería por el territorio y un año después llevaría a cabo un proceso de expansión a otros países. Tras un primer intento en Barcelona en 2016, Uber Eats empezaría a prestar servicios en Madrid en 2017. 
También en 2016 llegaría a España Stuart, más orientada en un principio a la mensajería, paquetería y comercio electrónico que al reparto de comida a domicilio. Durante todo este periodo el número de pedidos en las distintas plataformas, el volumen de negocio de estas empresas y, consecuentemente, el número de repartidores ha crecido de manera notable hasta la actualidad.

La aparición y crecimiento de este tipo de plataformas ha sido más o menos homogéneo y simultaneo en Europa y América. Sin embargo, en sus ritmos y evolución encontramos algunas diferencias. Así, es habitual que sean diferentes las plataformas que operan en los distintos países, que su implantación sea en momentos diferentes, la reacción de los mercados y las empresas predecesoras en la prestación de servicios ha sido desigual y la adaptación al marco jurídico y el mercado de trabajo de cada país ha debido responder a muy diferentes realidades y antecedentes. A pesar de esto, las problemáticas, escenarios laborales y debates generados en torno a la emergencia de estas formas de trabajo en el ámbito de las plataformas han respondido a dinámicas homogéneas y en un mismo sentido.

El rápido crecimiento de estas plataformas ha hecho que sea un objeto de estudio que ha ido ganando relevancia desde diferentes perspectivas. Esto se debe al creciente volumen de negocio y las rondas de financiación en las que se ha acumulado grandes cantidades de capital, pero también a una cada vez mayor visibilidad en las calles de repartidores y repartidoras, el mayor número de servicios que prestan las plataformas o los debates suscitados en torno a estas en la ciudadanía. Uno de los debates de mayor importancia ha sido la transformación de las formas de trabajo y los nuevos escenarios en los mercados laborales (Fleming, 2017). La importancia del trabajo en este debate se debe a las consecuencias como la aparición de nuevas formas de economía y modelos empresariales, las implicaciones de la popularización de determinadas formas de relaciones laborales -como la simulación de trabajo por cuenta propia- y sus consecuencias sobre los seguros sociales y los sistemas de protección, la situación jurídica de estas formas de trabajo o la organización colectiva y el sindicalismo en estas plataformas. Ante esto, ¿estamos ante un cambio en las formas del trabajo? Y ¿qué dinámicas hacen plantearnos si se presenta una tendencia al cambio en un sentido u otro? Así, en este apartado se aborda desde el caso de estudio del trabajo en las principales plataformas de reparto de comida y mensajería en España algunos elementos clave que señalarían ciertas dinámicas y mecanismos. Estos mecanismos y dinámicas serían homogéneos y diferenciados de otros procesos en el ámbito laboral aparecidos en la última década como formas de precarización o individualización del trabajo al margen de las plataformas digitales, pero también presente en los mercados laborales contemporáneos.

Así, en la misma formulación de la concepción del trabajo en estas plataformas residirían un modelo de negocio que acarrea consigo una lógica y una concepción social de las relaciones laborales y el trabajo en las sociedades contemporáneas; la constitución de un discurso concreto sobre el trabajo enmarcado en las lógicas neoliberales de individualización. Sin embargo, es importante tener en cuenta que estas tendrían un carácter dinámico dado que se enfrentan 
a realidades laborales y marcos legislativos complejos y cambiantes que frenan o permiten la implantación y el desarrollo de estas formas de trabajo -como ha ocurrido con las sentencias judiciales o los desarrollos legislativos recientes-, en los que por tanto, su proceso de implantación es complicado y poco uniforme (Rahman y Thelen, 2019).

Aquí se abordan desde tres grupos diferenciados algunas de las dinámicas que caracterizan al trabajo en las plataformas de reparto de comida y mensajería: la profundización y renovación de determinados mecanismos de control, disciplina y vigilancia en el trabajo; el tiempo como elemento de ordenación en las plataformas digitales; y la profundización de la tendencia a la individualización en el trabajo. Aunque no son las únicas que se dan en el trabajo de plataformas, estas son aquellas que identificamos con un carácter transversal, homogéneo y constante en las distintas plataformas. Así, vienen caracterizando estas formas de trabajo y sugieren la introducción y profundización de algunos de los aspectos de la transformación del trabajo contemporáneo.

Asimismo, es necesario tener en cuenta que al trabajo en las plataformas y, en particular, las de reparto de comida y mensajería, les ha venido caracterizando una constante actualización de las directrices y acuerdos específicos del trabajo. En otras palabras, quienes trabajan en estas plataformas se ven sometidos $-\mathrm{y}$ así lo ponen de manifiesto en las entrevistas realizadas- a un frecuente cambio de la condiciones y organización del trabajo, como son las cuantías de los pagos de los diferentes servicios, las formas de distribución de horarios o las maneras de comunicación con la empresa, entre otros. Estos cambios, frecuentes y poco previsibles en los aspectos específicos de las condiciones concretas del trabajo llevados a cabo de manera unilateral por parte de las empresas de plataforma constituyen en sí mismo también un rasgo de este tipo de trabajo.

\subsection{Mecanismos de control, disciplinamiento y vigilancia del trabajo}

En las formas de trabajo aparecidas en los últimos años uno de los elementos que se ha visto sujetos a cambios han sido las formas de vigilancia, control y disciplina. Esto sería importante en el ámbito de las plataformas, ya que es caracterizador de las relaciones que se establecen entre las empresas y quienes trabajan en el reparto. Las nuevas formas de vigilancia, control y disciplina se manifiestan así de distintas maneras, debido en buena medida a la relación laboral atípica y difusa que se establece entre repartidores y las empresas.

Uno de los rasgos de esta relación es la indeterminación y confusión en torno a quién o qué establece y controla la organización del trabajo. Desde la planificación de turnos y horarios, hasta ante quién se debe dar respuesta y cumplir exigencias de productividad. Esto se ve reflejado en el discurso de los repartidores en lo relativo a la ausencia de la figura del "jefe" ante el que se deben dar cuentas. Así, la ausencia de superiores directos en términos tradicionales es un carácter constante en los discursos de quienes trabajan en estas plataformas. Habitualmente los entrevistados señalaban que este había sido un aliciente y 
atractivo para elegir esta forma de trabajo. Sin embargo, esta es una situación que puede resultar paradójica. Para una parte de quienes trabajan en estas plataformas la ausencia de la figura de jefes o superiores infiere cierta libertad y autonomía en el desarrollo de su trabajo con respecto a otras formas de trabajo y experiencias laborales previas. Esto confiere al "algoritmo" racionalidad, objetividad e imparcialidad de carácter técnico sin prestar atención al carácter humano y arbitrario que atraviesa al algoritmo en su diseño (Griesbach, et al., 2019: 7). Pero observamos que los mecanismos que efectúan control y vigilancia efectivos se han visto reforzados a través de los medios tecnológicos, como son: la geolocalización permanente, el conocimiento itinerario realizado, el registro de las horas de conexión o qué pedidos aceptan y rechazan, entre otros. Como reconocen buena parte de quienes trabajan en estas plataformas e, incluso, algunos de quienes tenían una percepción de mayor autonomía al no tener jefes directos.

También en este sentido, la pretendida autonomía que le es supuesta a una forma de organización del trabajo a través de un algoritmo, a priori neutral e imparcial, se ve contrapuesta a la incapacidad de toma de decisiones en aspectos concretos del trabajo diario. Algunos de los que señalan de manera habitual los repartidores son la incapacidad de fijar precios —una crítica habitual entre los entrevistados que dicen no ser realmente trabajadores por cuenta propia-, la capacidad limitada y sujeta a los distintos mecanismos de evaluación para la selección de horarios o las implicaciones a la hora de elegir qué servicios se realizan y cuáles no, lo que venía repercutiendo negativamente en su calificación.

Estas calificaciones juegan un papel fundamental, ya que van a ser determinantes a la hora de la asignación del trabajo en distintas dimensiones: cuántas y cuáles horas se trabaja o qué pedidos concretos son asignados dependiendo de la plataforma. Esto es importante para entender que entran en competencia otros trabajadores, pero también consigo mismos. La puntuación se les concede a estos trabajadores a través de diferentes criterios que van desde la puntualidad, comentarios de los restaurantes o las calificaciones de los consumidores, entre otros. Esta puntuación les sitúa en unos ránquines en los que según su posición tienen, principalmente, la capacidad de seleccionar los horarios que trabajan. Esto genera una medida de presión sobre los trabajadores en dos dimensiones de la competencia. Una, con respecto a ellos mismos, en la que siempre van a querer trabajar las horas de más pedidos y de la mejor manera posible para poder seleccionar los horarios que desean o, al menos, los que consideran mejores de los que ha ido eligiendo el resto de trabajadores que estaban en posiciones más altas de estos ránquines. Una segunda, de competición con respecto al resto de trabajadores en la que la comparación podríamos considerar invisible, ya que solo conocen su propia puntuación, pero no la de sus compañeros. Como han señalado en las entrevistas estos repartidores, esto supone una gran presión sobre su trabajo. Observamos que este mecanismo actúa presionando con unos estándares de calidad y exigencia basados en las expectativas de la calidad del trabajo del resto de compañeros y que, por lo tanto, llevan a estos trabajadores a tener que mejorar su rendimiento sin tener una presión directa desde la empresa, solo 
su esperanza de tener la posibilidad de situarse en una posición relativa mejor que la de otros compañeros.

\subsection{Las plataformas de reparto como vía de acceso al mercado laboral, el tiempo a debate}

Las condiciones y la velocidad de expansión de este sector han implicado que sea una vía accesible al mercado laboral, siendo la primera o la única manera de inclusión en este para un gran número de personas. Esto se ve reflejado en quienes tienen un primer contacto con el mercado laboral (jóvenes) o quienes tienen limitadas otras posibilidades (población migrante y personas afectadas por concatenación de largos periodos de desempleo, como es el caso de los más afectadas por la crisis de 2008), como explican los entrevistados. Lo que también algunos de los entrevistados que llevan trabajando más tiempo en este ámbito señalan que lleva a una desprofesionalización del trabajo de reparto.

Esta facilidad de acceso se debe a la voluntad de las plataformas de tener una numerosa incorporación de personas como personal disponible para prestar servicios, sobredimensionando la fuerza de trabajo disponible en proporción a las necesidades del mercado. El no contraer apenas responsabilidades económicas con respecto a la disposición de una gran cantidad de trabajadores -a quienes solo les es pagado el trabajo realizado y no tener las obligaciones tributarias propias del trabajo por cuenta ajena-, permite a las empresas de plataforma incorporar a trabajadores sin formación, ni experiencia previa en el sector. Como así señalan casi todos los entrevistados, quienes expresaban que este había sido su primer contacto con el trabajo de reparto, mensajería, transporte o logística.

Aunque las plataformas de reparto presentaban estas formas de trabajo como una forma de complementar los ingresos de otra fuente principal o de obtener unos ingresos extras realizando una actividad atractiva (desplazarse y circular en bicicleta), esto no ha sido así (Fernández-Trujillo, 2020). Como se señala el perfil y la motivación de quienes han venido a incorporarse en esta actividad ha cambiado y se ha precarizado. Cada vez un número mayor de personas tienen como única actividad y exclusiva fuente de ingresos el trabajo en las plataformas digitales, estimándose que en 2017 para el 48\% de quienes trabajaban para plataformas estas eran su única fuente de ingresos (Berg, et al., 2019: 43-44) y la totalidad de los entrevistados señalaban que era la única o casi exclusiva vía de ingresos mientras trabajaban en estas plataformas. Las implicaciones sobre las condiciones de vida de esta forma de trabajo para obtener ingresos suficientes, el precio bajo del servicio de reparto impuesto por las empresas de plataforma y la falta de responsabilidad en términos tributarios de estas empresas en lo que respecta a pagos a la seguridad social y seguros para trabajadores hace que sea una forma de trabajo poco confortable, garantista y estable. Así, para quienes buscan una fuente de ingresos complementaria o atractiva por la facilidad de acceder a ingresos, ven frustradas las expectativas creadas por la imagen de esta forma de trabajo creadas por las empresas de plataformas y acababan buscando 
otras alternativas laborales. En otras palabras, la necesidad de trabajar muchas horas por bajos ingresos, corriendo por cuenta de los repartidores y repartidores el pago de la cuota de autónomo y la seguridad social hace que el acceso a esta forma de trabajo sea elegido por quienes tienen pocas alternativas laborales y no por un sujeto trabajador de plataforma idealizado.

Ante esto, vemos que se deben dar unas condiciones para trabajar en estas plataformas. A través de las entrevistas realizadas se puede observar que el elemento común es el de la disposición a un empleo del tiempo determinado, siendo esta una de las cuestiones clave que definiría el trabajo en las plataformas de trabajo de reparto y mensajería. Así, en este ámbito deberíamos preguntarnos a propósito de una cuestión clave en la concepción misma del trabajo en las plataformas de reparto. como es la puesta del tiempo a disposición de la empresa. Si bien, no tendríamos problema en percibir que el tiempo efectivo en el que se está llevando a cabo un servicio es parte de una fuerza de trabajo que será retribuida, quienes trabajan en estas plataformas explican que buena parte del tiempo que dedican están a la espera de pedidos no está remunerado. En este sentido, es fundamental saber que - de manera general, a excepción del caso de algunas plataformas en algunos periodos concretos - lo que es pagado es el servicio y no la hora trabajada, por lo tanto, todo el tiempo de espera deben estar conectados en la zona de reparto que tienen asignada y es tiempo por el que no se percibe ninguna retribución. Es fundamental en este sentido que lo que están ofreciendo quienes trabajan para estas plataformas no es, como en otros ámbitos, fuerza de trabajo a la que se le pone precio en función de la experiencia o formación, sino que su acogida en estas plataformas viene dada por la disposición de tiempo y, de manera complementaria, el esfuerzo físico y emplear horas "inactivas" en estar en la calle. En este sentido, señalaban que buena parte de su actividad era estar conectados a la aplicación y esperando a recepción de pedidos, sin ser remunerados por ello.

De la misma manera, estos trabajadores, en plataformas como Glovo, emplearían una gran cantidad de horas en la revisión de la aplicación para poder seleccionar sus horas de trabajo. La plataforma abre nuevas franjas horarias en las que trabajar y los trabajadores deben ser los primeros en seleccionarlas para poder trabajarlas, ya que el número de repartidores que pueden estar activos simultáneamente en un horario determinado es limitado - a diferencia de otras como Uber Eats, en las que los repartidores se pueden conectar y desconectar para la recepción de tareas de manera más o menos libre-. Esto es algo que los repartidores no solo hacen en las horas en las que se está en la calle desarrollando la actividad, esos tiempos en los que se está a la espera de pedidos, sino que también se hace en el tiempo libre, por lo que las fronteras del tiempo de trabajo se ven difuminadas. 


\subsection{La individualización de las relaciones laborales}

Como se ha venido señalando en los últimos años, las formas laborales propias de las transformaciones del trabajo a través la economía de plataformas, la gig economy y ciertas formas de economía digital han profundizado la tendencia hacia la individualización de las relaciones laborales en diferentes sentidos.

En términos jurídico-legales estos procesos de individualización se han consolidado a través de la ausencia de un marco jurídico propio y, lo que Baylos Grau llama, la "huida del derecho del trabajo" (Todolí, 2017). Esto ha permitido a las empresas plantear que las formas de relación laboral que venían generando tienen un carácter novedoso y ausente de marco legal a la que estar sujeto. Así, habían articulado estas relaciones con quienes trabajan en sus plataformas como terceros que prestaban servicios (trabajadores autónomos) y no como trabajadores asalariados con contrato laboral. Esto implica la ausencia de un marco jurídico común para todos estos trabajadores y la carencia de acceso convenios y negociaciones de carácter colectivo, llevando a una pérdida de la capacidad de organización y sindicación reconocida. Así, sería una vulneración de los derechos de los trabajadores como se ha señalado en sucesivas sentencias que se han dado en España en las que se reconocerían todos los derechos propios de los trabajadores, entre ellos los de representación y negociación colectiva. A pesar de la relevancia de esta cuestión no nos gustaría detenernos en esta dimensión jurídico-legal al haber sido ampliamente analizada.

Sin embargo, querríamos prestar atención a otros mecanismos que implican un distanciamiento de quienes trabajan en estas plataformas. La individualización no se ve solo reflejada en el aislamiento de quienes trabajan en lo relativo a la comunicación y relación con la empresa, sino también con el resto de personas que trabajan en las mismas plataformas de reparto, como hemos visto en el caso de los sistemas de calificación. Entre los discursos de quienes trabajan en estas plataformas encontramos habitualmente la percepción de que las empresas de plataforma han tomado medidas para que la comunicación y los espacios comunes para quienes trabajaban para ellas se vean reducidos. Ejemplo de estas medidas son la necesidad de estar circulando constantemente para tener mayor probabilidad de recibir pedidos y suprimir los espacios compartidos como los "centroides", antiguos puntos de espera para las zonas de asignación de pedidos. Como muestra de la importancia de estos puntos de encuentro, los centroides fueron donde se empezaron a fraguar informalmente las primeras movilizaciones y respuestas al empeoramiento de las condiciones en 2017.

El aislamiento e individualización en las plataformas se ve alimentado por el elevado número de rotación de personas que trabajan en estas plataformas, siendo una actividad en la que no se permanece durante largos periodos habitualmente. A pesar de que en los discursos de repartidores se pone de manifiesto que es claro el nivel de compañerismo, el constante cambio de personas y el poco tiempo que permanecen en el desarrollo de esta actividad de manera generalizada impide el establecimiento de redes permanentes y fortalecidas que profundicen en las dinámicas de solidaridad, apoyo y estrategias comunes frente 
a la empresa que permitiría el establecimiento de estructuras organizativas de los trabajadores más sólidas. Una muestra de esto, es que buena parte de las personas que están organizadas para la mejora de las condiciones en las plataformas digitales no siguen trabajando para estas empresas.

En este sentido, es habitual encontrar entre los discursos que hay una promoción del distanciamiento y la competencia entre compañeros a la hora de poder acumular horas de trabajo, elegir los mejores horarios, elegir los mejores servicios, etc. mientras que la relación y el compañerismo entre quienes trabajan en estas plataformas es puesto en valor. A través chats de grupos de Whatsapp o Telegram o en los pocos espacios en los que pueden encontrarse de manera breve, como las puertas de los restaurantes, explican los entrevistados que se dan muestras de solidaridad y compañerismo, cubriendo en buena medida las carencias de la organización del trabajo por parte de las plataformas. Ejemplos de esto serían desde el funcionamiento específico de algunos aspectos de las plataformas, los paquetes de horas que son abiertos para cogerlos o la ayuda ante eventuales accidentes.

Los sistemas de selección de horarios y sus problemáticas, como han sido expuestos anteriormente, también repercutirían en la individualización de estos trabajadores. La competencia fomentada con respecto al resto les haría estar solos ante la aplicación y su posición, ya que la mejor puntuación de sus compañeros va en detrimento de su posición relativa.

\section{CONCLUSIÓN}

Este artículo analiza de forma comparada el tipo de trabajo y modo de producción que configuran las plataformas digitales, utilizando los casos de Airbnb y de las plataformas de reparto. En el caso de Airbnb, se trata de un tipo de trabajo y proceso productivo que: i. emerge sobre la esfera de la reproducción, lo que extiende y subsume la lógica del valor hacia nuevos ámbitos; ii. un tipo de trabajo invisibilizado, que no es reconocido como trabajo, aunque los anfitriones sí que consideran que están trabajando y desarrollen actividades propias del campo laboral (limpieza, entrega de llaves, etc); iii. y un tipo de trabajo empresarial, donde los anfitriones son los encargados de organizar el conjunto del proceso productivo, gestionando la oferta como si de una empresa se tratara, dotándola de valor, haciéndola competitiva e incluso ejecutando procesos de externalización y racionalización de su propio trabajo. De la misma manera, aunque el trabajo en las plataformas de reparto de comida y mensajería está sujeto a constantes cambios en algunas de sus condiciones específicas, a esta forma de trabajo le caracterizarían una serie de dinámicas: i. por un lado, una paradójica relación entre la percepción de autonomía y libertad como trabajadores autónomos contrapuesta a un fuerte despliegue de mecanismos de control y organización del trabajo por parte de las plataformas; ii. la disposición a emplear tiempo de trabajo no pagado, el cual sería un rasgo característico de quienes entran en el mercado de trabajo por esta vía; iii. las dinámicas de individualización y asi- 
lamiento en el desarrollo de esta actividad, pero también a nivel de la relación laboral entre repartidores y plataformas.

Así, si bien las plataformas digitales tienen un funcionamiento distinto, operan en distintos sectores y se basan en formas de trabajo fuertemente diferenciadas, podemos observar cómo comparten dinámicas que les son comunes. Estas formas de producción que derivan en inmediatez, inestabilidad e individualización y aislamiento en el ámbito del trabajo, estando bajo una misma dinámica de cambio en las lógicas del trabajo tal y como se había venido concibiendo. Las formas de trabajo en plataformas digitales juegan el mismo papel en el proceso de transformación del trabajo y el empleo en el marco del neoliberalismo.

En los dos casos analizados se puede observar cómo se dan situaciones semejantes. En ambos casos, estas plataformas han surgido como un nuevo ámbito de trabajo en expansión y empresas que han tenido una relevancia social y mediática notable en los últimos años. Las formas de trabajo generadas, sin embargo, están caracterizadas por un carácter atípico y no estándar con respecto a formas de trabajo más tradicionales del mercado laboral. Esto se ve reflejado en las paradojas que se dan en la organización del trabajo. Mientras que a quienes trabajan con estas plataformas se les supone una autonomía en la organización del trabajo, esta está fuertemente condicionada por las exigencias y estándares que establecen las plataformas (cómo deben entregarse y recogerse los pedidos, cómo debe darse la relación con los huéspedes, la cuantía de los pagos por los servicios de reparto, etc.). Así, se dan formulaciones difusas de la relación entre plataformas y quienes trabajan con ellas, lo que se ve claramente en el uso de la palabra "colaborador" para referirse a quienes trabajan con estas plataformas para evitar el reconocimiento del trabajo con esta categoría tan poco nítida. También, aunque las exigencias, organización del trabajo y condiciones es constante y semejante entre los que trabajan con ellas, la relación con estos trabajadores está individualizada y está imposibilitada una respuesta colectiva, al menos de manera reconocida por parte de las plataformas.

Por último, como se ha señalado, las fronteras del trabajo y del no-trabajo se ven fuertemente difuminadas en estas plataformas. Así, diferentes actividades relacionadas con el trabajo en la plataforma están disgregadas a lo largo del día, al margen de la principal actividad y del tiempo previsto para el trabajo, estando sujetos a estar conectados a la plataforma gestionando el trabajo que va a ser realizado (por ejemplo, en asignación de horas de trabajo en las plataformas de reparto o cuando los anfitriones reciben mensajes o socializan con los huéspedes en Airbnb). El resultado es que la actividad laboral se extiende sobre el conjunto de la vida, lo que invade las relaciones no laborales de la persona y subordinada y organiza las actividades vitales a la actividad laboral (aunque solo se remunere la actividad estrictamente productiva).

Como vemos, el trabajo en las plataformas es un ámbito en expansión en el que se vienen dando situaciones atípicas y novedosas en el ámbito laboral. Esto supone retos a la hora de comprender qué implican estos cambios sobre el trabajo en nuestras sociedades. Principalmente, porque las transformaciones del trabajo que se han visto en este artículo, cada vez tienen un peso mayor en 
nuestras sociedades y afectan a un número creciente de sectores económicos. En ese sentido, la "plataformización" en los procesos productivos también se podría entender como una forma de transformación del trabajo y de las relaciones laborales que incrementan el grado de explotación del trabajo por el capital. Por lo tanto, estas novedosas dinámicas, más que entenderse como propias de las plataformas digitales, se deben comprender como intrínsecas a la evolución del neoliberalismo.

\section{BIBLIOGRAFÍA}

AIRBNB. (2016a). Don't Go There. Live There. [Youtube]. Recuperado de https://www. youtube.com/watch?time_continue $=29 \& \mathrm{v}=$ bbdF8Muiwtk

AIRBNB. (2016b, noviembre 9). Sentir como en casa en cualquier rincón del mundo. Recuperado de https://blog.atairbnb.com/world-belonging-es/

ALONSO, L. E. (1994). Sujeto y discurso: el lugar de la entrevista abierta en las prácticas de la sociología cualitativa. Métodos y técnicas cualitativas de investigación en Ciencias Sociales. Madrid: Síntesis, 225-420.

ALONSO, L. E. (1998). La mirada cualitativa en sociología: una aproximación interpretativa (Vol. 218). Editorial Fundamentos.

ALONSO, L.E. (1999). Trabajo y ciudadanía. Estudios sobre la crisis de la sociedad salarial. Madrid: Trotta.

ALONSO, L. E., FERNÁNDEZ, C. J., Y IBÁÑEZ, R. (2016). De la moral del sacrificio a la conciencia de la precariedad: un análisis cualitativo de los discursos sobre la evolución de la crisis en España. Política y sociedad, 53(2), 353-379

ARRIBAS, J. M. (2013). Sociología del consumo e investigación de mercados. Una guía didáctica. Madrid: UNED.

BERG, J., FURRER M., HARMON, E., RANI, U. Y SILBERMAN, M. S. (2019). Las plataformas digitales y el futuro del trabajo. Cómo fomentar el trabajo decente en el mundo digital. Ginebra: OIT.

BOTSMAN, R., \& ROGERS, R. (2010). What's mine is yours: how collaborative consumption is changing the way we live. Collins London.

BROWN, W. (2015). Unduing the Demos: Neoliberalism's Stealth Revolution. In Zone Books. Zone Books. https://doi.org/10.21061/spectra.v7i1.128

CALDERÓN, J. Á., \& LÓPEZ: (2010). Transformaciones del trabajo e individualización de las relaciones laborales. La emergencia de nuevas formas de resistencia al trabajo. Estudios de La Fundación, 1-16.

CALLEJO, J. (2001). El grupo de discusión: introducción a una práctica de investigación. Barcelona: Ariel.

CAMPOS, R. L., \& HERNÁNDEZ, A. G. (2018). Expansion of temporality and erosion of the standard employment relationship in Spain: The emergence of a new paradigm of employment relationship? Cuadernos de Relaciones Laborales, 36(1), 3563. https://doi.org/10.5209/CRLA.59556

COMISIÓN EUROPEA (2016). Una Agenda Europea para la economía colaborativa. $<$ ec.europa.eu/DocsRoom/documents/16881/attachments/2/.../pdf>

COMOR, E. (2011). Contextualizing and critiquing the fantastic prosumer: Power, alienation and hegemony. Critical Sociology, 37(3), 309-327. 
CONDE, F., \& DEL ÁLAMO, G. (2009). Análisis sociológico del sistema de discursos (Vol. 43). Madrid: Centro de Investigaciones Sociológicas.

CRIADO, E. M. (1998). Los decires y los haceres. Papers, 56, 57-71.

CRIADO, E. M. (2014). Mentiras, inconsistencias y ambivalencias. Teoría de la acción y análisis de discurso. revista internacional de sociología, 72(1), 115-138.

DE RIVERA, J., LOPEZ, Á. J. G., \& CASSIDY: (2017). La economía colaborativa en la era del capitalismo digital. Revista de Estudios para el Desarrollo Social de la Comunicación, (15), 20-31.

DE STEFANO, V. (2015). The Rise of the 'Just-in-Time Workforce': On-Demand Work, Crowd Work and Labour Protection in the 'Gig-Economy. Comparative Labor Law \& Policy Journal, Bocconi Legal Studies Research Paper No. 2682602 , Available at http://dx.doi.org/10.2139/ssrn.2682602

DE STEFANO, V. (2018). Employment and Labour Market Policies Branch "Negotiating the algorithm": Automation, artifi cial intelligence and labour protection. 246. www.ilo.org/publns

DE VAUJANY, F. X., LECLERCQ-VANDELANNOITTE, A., \& HOLT, R. (2019). Communities Versus Platforms: The Paradox in the Body of the Collaborative Economy. Journal of Management Inquiry. https://doi.org/10.1177/1056492619832119

DEGRYSE, C. (2016). Digitalisation of the economy and its impact on labour markets. ETUI.

DENEGRI-KNOTT, J., \& ZWICK, D. (2012). Tracking prosumption work on eBay: Reproduction of desire and the challenge of slow re-McDonaldization. American Behavioral Scientist, 56(4), 439-458.

DRAHOKOUPIL, J., \& JEPSEN, M. (2017). The digital economy and its implications for labour. 1. The platform economy. Transfer, 23(2), 103-107. https://doi. org $/ 10.1177 / 1024258917701380$

ECKHARDT, G. M., HOUSTON, M. B., JIANG, B., LAMBERTON, C., RINDFLEISCH, A., \& ZERVAS, G. (2019). Marketing in the Sharing Economy. Journal of Marketing, 83(5), 5-27. https://doi.org/10.1177/0022242919861929

FAST, K., ÖRNEBRING, H., \& KARLSSON, M. (2016). Metaphors of free labor: a typology of unpaid work in the media sector. Media, Culture \& Society, 38(7), 963 978.

FERNÁNDEZ-TRUJILLO MOARES, F. (2020). Precariedad e inestabilidad: contradicciones en el trabajo en las plataformas de reparto de comida. Teknokultura. Revista de Cultura Digital y Movimientos Sociales, 17(1), 35-40. https://doi.org/10.5209/ tekn.65189

FLEMING: (2017). The Human Capital Hoax: Work, Debt and Insecurity in the Era of Uberization. Organization Studies, 38(5), 691-709. https://doi. org/10.1177/0170840616686129

FLORISSON, R., \& MANDL, I. (2018). Platform work: Types and implications for work and employment-Literature review Employment and working conditions of selected types of platform work. 1/132. www.eurofound.europa.eu

FUCHS, C. (2010). Labor in Informational Capitalism and on the Internet. The Information Society, 26(3), 179-196.

FUMAGALLI, A. (2007). Bioeconomía y capitalismo cognitivo. Traficantes de Sueños.

GAGO, V. (2015). La razón neoliberal: Economías barrocas y pragmática popular. Madrid: Traficantes de Sueños.

GALLAGHER, L. (2017b, febrero 15). Airbnb's Profits to Top \$3 Billion by 2020. Fortune. Recuperado de http://fortune.com/2017/02/15/airbnb-profits/ 
GIL, J. (2016). Economías colaborativas y crisis del capitalismo: un análisis a través de la prosumición. En Ciberpolítica: Gobierno abierto, redes, deliberación, democracia (pp. 167-188). Madrid: Instituto Nacional de Administración Pública.

GIL, J. (2018). Las múltiples formas de trabajo en las economías colaborativas y su regulación: El caso de «Airbnb». En Adrían Todolí y Macarena Hernández (Eds.): Trabajo en plataformas digitales: Innovación, derecho y mercado. Thompson-Reuters Aranzadi.

GIL, J. (2019). Crisis, innovación tecnológica y mercantilización neoliberal de la vida. El caso de Airbnb [Tesis doctoral]. Universidad Nacional de Educación a Distancia.

GORDO, Á., RIVER, J. DE, \& APESTEGUÍA, A. (2016). Facing the Challenge of Collaborative Consumption in Europe: A Time for Independent Metrics. 1-28.

GOTTLIEB, C. (2013). Residential Short-Term Rentals: Should Local Governments Regulate the'Industry'? Planning \& Environmental Law, 65(2), 4-9.

GRAHAM, M., HJORTH, I., \& LEHDONVIRTA, V. (2017). Digital labour and development: impacts of global digital labour platforms and the gig economy on worker livelihoods. Transfer, 23(2), 135-162. https://doi.org/10.1177/1024258916687250

HARTMANS, A. (2017, agosto 10). Airbnb now has more listings worldwide than the top five hotel brands combined. Business Insider. Recuperado de https://www.businessinsider.com/airbnb-total-worldwide-listings-2017-8?IR=T

HILL, S. (2015). Raw Deal: How the" Uber Economy” and Runaway Capitalism Are Screwing American Workers. St. Martin's Press.

HILL, D. W. (2019). The injuries of platform logistics. Media, Culture and Society. https://doi.org/10.1177/0163443719861840

HOWCROFT, D., \& BERGVALL-KÅREBORN, B. (2019). A Typology of Crowdwork Platforms. Work, Employment and Society, 33(1), 21-38. https://doi. org/10.1177/0950017018760136

HUMPHREYS, A., \& GRAYSON, K. (2008). The intersecting roles of consumer and producer: a critical perspective on co-production, co-creation and prosumption. Sociology Compass, 2(3), 963-980.

IBÁÑ̃̃EZ, J. (1979). Más allá de la Sociología. Madrid: Siglo XXI.

IBÁÑEZ, J. (1986). Perspectivas de la investigación social: el diseño en las tres perspectivas. En El análisis de la realizad social. Métodos y Técnicas de Investigación. Madrid: Alianza Universidad. Madrid.

IBÁÑ̃Z, J. (1991). El regreso del sujeto. Santiago: Amerindia.

KALAMAR, A. (2013). "Sharewashing is the New Greenwashing". OpEd News.

KÖRFER, A., \& RÖTHIG, O. (2017). Decent crowdwork - The fight for labour law in the digital age. Transfer, 23(2), 233-236. https://doi. org/10.1177/1024258917701364

GRIESBACH, K., REICH, A., ELLIOTT-NEGRI, L., \& MILKMAN, R. (2019). Algorithmic Control in Platform Food Delivery Work. Socius: Sociological Research for a Dynamic World, 5, 237802311987004. https://doi.org/10.1177/2378023119870041

LARA, Á. L. (2013). Realidades de la ficción. Bioproducción y trabajo cognitivo en la fábrica televisiva. La escritura de series de televisión en Epsaña: cualidades y condiciones de los trabajos y los trabajadores. Universidad Complutense de Madrid, Madrid.

LAVAL, C. Y DARDOT: (2013). La nueva razón del mundo. Ensayo sobre la sociedad neoliberal. Barcelona: Gedisa Editorial. Miguélez, 2018;

OROZCO, A. (2014). Subversión feminista de la economía. Aportes para un debate sobre el conflicto capital-vida. Traficantes de sueños., (3). 
OSKAM, J., \& BOSWIJK, A. (2016). Airbnb: the future of networked hospitality businesses. Journal of Tourism Futures, 2(1), 22-42.

QUATTRONE, G., PROSERPIO, D., QUERCIA, D., CAPRA, L., \& MUSOLESI, M. (2016). Who benefits from the sharing economy of Airbnb? En Proceedings of the 25th International Conference on World Wide Web (pp. 1385-1394). International World Wide Web Conferences Steering Committee.

RAHMAN, K. S., \& THELEN, K. (2019). The rise of the platform business model and the transformation of twenty-first-century capitalism. Politics and Society, 47(2), 177-204. https://doi.org/10.1177/0032329219838932

RAVENELLE, A. J. (2017). Sharing economy workers: selling, not sharing. Cambridge Journal of Regions, Economy and Society, 10(2), 281-295.

REY: J. (2012). Alienation, exploitation, and social media. American Behavioral Scientist, 56(4), 399-420.

RIFKIN, J. (2014). La sociedad de coste marginal cero: el internet de las cosas, el procomún colaborativo y el eclipse del capitalismo. Barcelona: Grupo Planeta (GBS).

RITZER, G., \& JURGENSON, N. (2010). Production, Consumption, Prosumption The nature of capitalism in the age of the digital 'prosumer'. Journal of consumer culture, 10(1), 13-36.

SADIN, É. (2018). La silicolonización del mundo. La irresistible expansión del liberalismo digital. Buenos Aires: Caja Negra

SANTOS, A. Y MARTÍN: (2012). La juventud española en tiempos de crisis. Paro, vidas precarias y acción colectiva. Socio- logía del trabajo, 75, 93-110.

SCHOLZ, T. (2016). Platform Cooperativism. Challenging the Corporate Sharing Economy. New York: Rosa Luxemburg Stiftung.

SRNICEK, N. (2018). Capitalismo de plataformas. Buenos Aires: Caja Negra.

SCHOR, J. B., \& ATTWOOD-CHARLES, W. (2017). The "sharing" economy: labor, inequality, and social connection on for-profit platforms. Sociology Compass, 11(8), 1-16. https://doi.org/10.1111/soc4.12493

SCHOR, J. (2017). Does the sharing economy increase inequality within the eighty percent?: findings from a qualitative study of platform providers. Cambridge Journal of Regions, Economy and Society, 10(2), 263-279.

SLEE, T. (2015). What's Yours Is Mine. OR Books, New York-London.

SPERLING, G. (2015). How Airbnb combats middle class income stagnation. Sperling Economic Strategies.

SUNDARARAJAN, A. (2016). The sharing economy: The end of employment and the rise of crowd-based capitalism. Mit Press.

TERRANOVA, T. (2000). Free labor: Producing culture for the digital economy. Social text, 18(2), 33-58.

TODOLÍ-SIGNES, A. (2017). The 'gig economy': employee, self-employed or the need for a special employment regulation? Transfer, 23(2), 193-205. https://doi. org/10.1177/1024258917701381

VALLAS, S. P. (2019). Platform Capitalism: What's at Stake for Workers? New Labor Forum, 28(1), 48-59. https://doi.org/10.1177/1095796018817059

VAN DOORN, N. (2017). Platform labor: on the gendered and racialized exploitation of low-income service work in the 'on-demand' economy. Information Communication and Society, 20(6), 898-914. https://doi.org/10.1080/1369118X.2017.1294194

VANDAELE, K. (2018). Will Trade Unions Survive in the Platform Economy? Emerging Patterns of Platform Workerss Collective Voice and Representation in Europe. SSRN Electronic Journal. https://doi.org/10.2139/ssrn.3198546 
VEEN, A., BARRATT, T., \& GOODS, C. (2019). Platform-Capital's 'App-etite' for Control: A Labour Process Analysis of Food-Delivery Work in Australia. Work, Employment and Society. https://doi.org/10.1177/0950017019836911

ZERVAS, G., PROSERPIO, D., \& BYERS, J. W. (2014). The rise of the sharing economy: Estimating the impact of Airbnb on the hotel industry. Journal of Marketing Research. Recuperado de http://journals.ama.org/doi/abs/10.1509/jmr.15.0204 